Corti Varela, Justo: “Implicaciones de la reciente autorización de comercialización para el consumo humano del salmón modificado genéticamente”. IUS ET SCIENTIA, 2015, Vol. 1, No. 1, pp. 93-109.

DOI: http://dx.doi.org/10.12795/IETSCIENTIA.2015.i01.07

\title{
Implicaciones de la reciente autorización de comercialización para el consumo humano del salmón modificado genéticamente.
}

\section{Implications of the recent marketing authorization for human consumption of genetically modified salmon}

Dr. Justo Corti Varela. Prof. Colaborador Doctor de la Universidad CEU San Pablo. Prof. visitante de la Université Nanterre (Paris X) jlcorti@ceu.es

Resumen. En este artículo se estudia la autorización del salmón AquAdvantage como un evento clave en el proceso de toma de decisiones sobre productos al constituir el primer animal modificado genéticamente en ser autorizado para comercializarse con fines de alimentación humana. Esta autorización abrirá la puerta a nuevas autorizaciones que, sin duda, cambiarán por completo el campo de las relaciones entre derecho y ciencia, al menos en cuanto a biotecnología se refiere. Sin embargo, para lograr la aceptación del público es necesario demostrar que la agencia encargada de la evolución de riesgos, tanto sanitarios como medioambientales, ha realizado un trabajo pormenorizado y completo, con suficiente transparencia, lo que no parece ser el caso. Una medida que podría limitar el efecto de este rechazo de la sociedad a estos productos transgénicos sería el establecimiento de un etiquetado obligatorio sobre el salmón modificado genéticamente, similar al que impera en la UE para las plantas transgénicas.

Abstract. This article focus on the authorization for AquAdvantage salmon a key event in the process of making decisions about animal products genetically modified to be allowed to be marketed for human consumption purposes. This approval opens the door to new authorizations and it undoubtedly changes completely the relations between law and science, at least as far as biotechnology is concerned. However, to achieve public acceptance is necessary to show that, both health and environmental agency responsible for the evaluation of risks has made a detailed and comprehensive work, with sufficient transparency, which does not seem to be the case. A move that could limit the effect of this rejection of society to these products would be the establishment of a mandatory labeling on genetically modified salmon, similar to that prevailing in the EU for transgenic plants.

Palabras clave. Productos trasngénicos - Regulación normativa europea de OGMs Etiquetado y riesgos para la salud

Key words. GM products - Regulation European regulation of GMOs - Labelling and health risks

\section{Introducción}

Los salmones son peces que pasan la mayor parte de su vida en el mar y, al llegar el momento de reproducción vuelven para desovar al río de agua dulce, normalmente causes de montaña y de deshielo, que un día los vio nacer. Esta cualidad que intriga a científicos, los cuales todavía no han sido capaces de descifrar cómo hacen 
Corti Varela, Justo: “Implicaciones de la reciente autorización de comercialización para el consumo humano del salmón modificado genéticamente”. IUS ET SCIENTIA, 2015, Vol. 1, No. 1, pp. 93-109.

DOI: http://dx.doi.org/10.12795/IETSCIENTIA.2015.i01.07

esos peces adultos para identificar exactamente el lugar en cuestión entre los muchos otros cursos de agua no será más el elemento más intrigante de estos animales acuáticos. Recientemente la FDA (Food and Drugs Administration), la agencia americana con potestad para autorizar los alimentos para consumo humano en los Estados Unidos, acaba de autorizar el primer animal genéticamente modificado para uso alimenticio. Este hecho constituye, sin duda, un antes y un después en la regulación jurídica de uno de los campos más controvertidos de la ciencia moderna. El éxito de esta apuesta que está realizando la administración americana, mensurable no solo par la efectiva confirmación en condiciones reales y de uso masivo de la seguridad del producto y de la efectividad de los protocolos de prevención pero también en la aceptación del mismo por parte de los consumidores marcará el curso de la regulación de animales transgénicos y el grado de certitud que exigirán los reguladores para futuras autorizaciones.

A finales de los ochenta, la empresa AquaBounty Technologies, comenzó a desarrollar el salmón AquAdvantage, un tipo de salmón modificado genéticamente para que produjese durante todo el año una hormona del crecimiento, similar a la insulina, en vez de hacerlo sólo en los meses cálidos como hacen los salmones convencionales. Durante el invierno, coincidiendo con la época del año en que normalmente escasean sus presas y las bajas temperaturas exigen un uso más racional de los recursos proteicos y grasos, los salmones dejan de producir dicha hormona y, así, ralentizan su crecimiento. En un contexto de cría en cautividad con fines comerciales, donde los especimenes son alimentados con piensos de forma regular, no se justificaría el uso de dicho mecanismo fisiológico. Sin embargo, y a pesar de estar sometidos a mecanismos de selección genética, los criadores de salmones no habían logrado impedir esta programación genética. Es precisamente este escollo lo que quisieron sortear los biólogos de AquaBounty cuando decidieron combinar dos genes, una proteína de la hormona del crecimiento del salmón real del Pacífico o Chinook con secuencias de ADN del la proteína anticongelante de una anguila oceánica que vive en aguas árticas, e insertar el resultado en el genoma del salmón atlántico de uso comercial. La modificación actúa como un interruptor que mantiene activa la hormona del crecimiento 
Corti Varela, Justo: “Implicaciones de la reciente autorización de comercialización para el consumo humano del salmón modificado genéticamente”. IUS ET SCIENTIA, 2015, Vol. 1, No. 1, pp. 93-109.

DOI: http://dx.doi.org/10.12795/IETSCIENTIA.2015.i01.07

durante todo el año y, así, disminuye a la mitad el tiempo en que tardan los salmones en llegar a adultos y, con ello, a un tamaño que permita su venta comercial ${ }^{1}$.

En este artículo $^{2}$ no nos centraremos sólo en explicar la evolución del proceso de autorización, y los procedimientos y requisitos a que se sometió el producto para evaluar sus riesgos; sino también analizaremos el grado de transparencia que los organismos de control han querido darle al mismo y las posibilidades reales de participación que ha tenido la sociedad civil en el proceso de toma de decisiones. Este último elemento resulta esencial para poder predecir, al menos en términos generales, el grado de aceptación que podrá tener el producto, así como para valorar la oportunidad de introducir medidas complementarias de información a los consumidores para evitar que fallos en la transmisión pudiesen producir efectos contraproducentes en la percepción del riesgo.

\section{Un poco de historia}

El salmón AquAdvantage no es el primer animal transgénico. De hecho, uno de los primeros grandes éxitos comerciales de la biotecnología fue el Oncomouse, un ratón diseñado por investigadores de la Universidad de Harvard a comienzos de la década de los ochenta que tenía activado un gen específico para desarrollar cáncer de mama. Así, el ratón de laboratorio tenía tal propensión para el cáncer que era perfecto para el estudio de dicha enfermedad y de tratamientos y fármacos para tratarla.

Tampoco debemos olvidar la mascota GloFish, un pez tropical para acuarios que cambia de color cuando se lo iluminaba con luz ultravioleta ${ }^{3}$

Según Vàzquez-Salat et al. ${ }^{4}$, los animales modificados genéticamente pueden clasificarse según su uso en:

\footnotetext{
${ }^{1}$ George Kimbrell y Paige Tomaselli “A 'fisheye' lens on the technological dilemma: The Specter of Genetically Engineered Animals” 18 Animal Law 75 (2011), p. 75 et sqq., en p. 76.

${ }^{2}$ Algunos elementos fueron tratados por el mismo autor antes de que la FDA tomara la decisión definitiva de autorizar el salmón AquAdvantage. Ver Justo Corti Varela " FDA is ready to Authorize GM Salmons but is the Market ready for them? European Journal of Risk Regulation 4 (2013), p. 521 et sqq.

${ }^{3}$ El GloFish es, hasta la aprobación del salmón transgénico, el único animal genéticamente modificado que estaba disponible en el mercado. Sin embargo, seguramente por su uso confinado y su destino no alimentario, nunca fue sometido a un proceso de aprobación para uso comercial. Ver Rebecca M. Bratspies, “Glowing in the Dark: How America’s First Transgenic Animal Escaped Regulation”, 6 Minnesota Journal of Law, Science \& Technology (2005), p. 457, et sqq., esp. p. 475-77.

${ }^{4}$ Núria Vàzquez-Salat, Brian Salter, Greet Smets, Louis-Marie Houdebine “The current state of GMO governance: Are we ready for GM animals?” 30 Biotechnology Advances (2012) pp. 1336 et sqq., esp. en p. 1337.
} 
Corti Varela, Justo: “Implicaciones de la reciente autorización de comercialización para el consumo humano del salmón modificado genéticamente”. IUS ET SCIENTIA, 2015, Vol. 1, No. 1, pp. 93-109.

DOI: http://dx.doi.org/10.12795/IETSCIENTIA.2015.i01.07

i) aquellos destinados a la investigación básica, como por ejemplo el ya citado Oncomouse.

ii) los usados para trasplantes entre diferentes especies o xenotransplantes en donde la modificación genética, generalmente en cerdos, impide o reduce las posibilidades de rechazo.

iii) los bio-reactores, es decir, los que se modifican para que segreguen, por ejemplo leche, con unas especiales características, tal como produce el conejo Ruconest.

iv) los destinados a mascotas, como el GloFish.

v) y, finalmente, los modificados para aumentar su productividad, como el caso del salmón AquAdvantage

La mayoría de los animales modificados genéticamente son ratones o conejillos de indias usados para investigación básica o bio-reactores, es decir, para un uso confinado en laboratorios. A pesar de la importancia de ciertas modificaciones, paradójicamente, esos usos no generan un fuerte rechazo social ya que se considera, primero, que el confinamiento reduce significativamente los riesgos medioambientales ya que un escape fortuito sería muy excepcional; y en segundo término porque en la balanza de riesgos y beneficios los usos medicinales o de investigación se consideran claramente más importantes que los alimenticios o recreativos ${ }^{5}$.

De hecho el salmon AquAdvantage es el primer animal modificado genéticamente para fines alimenticios en vencer estas reticencias, por lo que se esperar que abra la puerta para otros animales biotecnológicos con usos similares en el futuro.

Sin embargo, el camino no ha sido fácil para AquaBounty Technologies. El proceso de autorización ha demorado más de dos décadas, ya que iniciado en 1993 no fue hasta 2015 en que finalmente el salmón transgénico ha sido autorizado para consumo humano ${ }^{6}$. El procedimiento había alcanzado un paso significativo cuando en

\footnotetext{
${ }^{5}$ Sheryl Lawrence, "What Would You Do with a Fluorescent Green Pig?: How Novel Transgenic Products Reveal Flaws in the Foundational Assumptions for the Regulation of Biotechnology”, 34 Ecology Law Quarterly (2007) pp. 201 et sqq., esp. en pp. 256-59.

${ }^{6}$ La autorización fue expedida por carta de fecha 19 de noviembre de 2015 (NADA 141-454) sobre la base de la Evaluación (definitiva) de riesgos medioambientales de fecha 12 de noviembre de 2015 y el FONSI (definitivo) de la misma fecha. Ver Center for Veterinary Medicine - FDA, "Freedom of information summary - NADA 141-454” 12 de noviembre de 2015. De ahora en adelante haremos referencia a este informe como-FDA 2015. Disponible en internet en el siguiente enlace:
} 
Corti Varela, Justo: “Implicaciones de la reciente autorización de comercialización para el consumo humano del salmón modificado genéticamente”. IUS ET SCIENTIA, 2015, Vol. 1, No. 1, pp. 93-109.

DOI: http://dx.doi.org/10.12795/IETSCIENTIA.2015.i01.07

septiembre de 2010, siguiendo al igual que en el caso de la biotecnología vegetal el principio de la equivalencia sustancial, la FDA había considerado que el salmón modificado genéticamente "is as safe as food from conventional Atlantic salmon”7. Después de superar un período de consultas públicas y un año de deliberaciones, en mayo de 2012 la propia FDA había emitido una evaluación de impacto ambiental (provisional) proponiendo las condiciones para autorizar su $\mathrm{uso}^{8} \mathrm{y}$ unas primeras "Finding of No Significant Impact (FONSI)" de carácter provisional ${ }^{9}$. Entre estas primeras decisiones provisionales y las definitivas de este año hubo un segundo período de consultas públicas en las que la FDA recibió más de 36000 comentarios, en su amplia mayoría contra la autorización. La decisión que acabamos de conocer se encontraba pendiente desde abril de 2013, por lo que no se puede decir que la administración americana haya tomado una medida precipitada.

\section{Beneficios y riesgos del salmón AquAdvantage}

Tal como se adelantó en la introducción, el Salmón AquAdvantage crece dos veces más rápido que sus homólogos convencionales. Por lo tanto, el principal beneficio ligado a su implantación es la reducción de los costes de cría. Reducir los tiempos de

http://www.fda.gov/downloads/AnimalVeterinary/DevelopmentApprovalProcess/GeneticEngineering/Ge neticallyEngineeredAnimals/UCM466215.pdf (último acceso 29 de noviembre de 2015). Ver también Center for Veterinary Medicine - FDA, “AquAdvantage Salmon Environmental Assessment” 12 de noviembre de 2015. De ahora en adelante haremos referencia a este informe como -Evaluación ambiental definitiva 2015. Disponible en internet en el siguiente enlace: http://www.fda.gov/downloads/AnimalVeterinary/DevelopmentApprovalProcess/GeneticEngineering/Ge neticallyEngineeredAnimals/UCM466218.pdf (último acceso 29 de noviembre de 2015). Asimismo consultar Center for Veterinary Medicine - FDA, “AquAdvantage Salmon FONSI (Finding of No Significant Impact)” 12 de noviembre de 2015. De ahora en adelante haremos referencia a este informe como -FONSI definitivo 2015. Disponible en internet en el siguiente enlace: http://www.fda.gov/ downloads/AnimalVeterinary/DevelopmentApprovalProcess/GeneticEngineering/GeneticallyEngineered Animals/UCM466219.pdf (último acceso 29 de noviembre de 2015).

${ }^{7}$ Center for Veterinary Medicine - FDA, “Briefing Packet: AquAdvantage Salmon” 20 September 2010, en la p. 71. De ahora en adelante haremos referencia a este informe como FDA 2010. Disponible en internet en el siguiente enlace: http:/www.fda.gov/downloads/ AdvisoryCommittees/CommitteesMeetingMaterials/VeterinaryMedicineAdvisoryCommittee/UCM22476 2.pdf (ultimo acceso 29 de noviembre de 2015).

${ }^{8}$ Center for Veterinary Medicine - FDA, "Draft Environmental Assessment: AquAdvantage Salmon” 4 de mayo de 2012. De ahora en adelante haremos referencia a este informe como -Evaluación ambiental provisional 2012. Disponible en internet en el siguiente enlace: http://www.fda.gov/downloads/AnimalVeterinary/DevelopmentApprovalProcess/GeneticEngineering/Ge neticallyEngineeredAnimals/UCM333102.pdf (último acceso el 29 de noviembre de 2015).

${ }^{9}$ Center for Veterinary Medicine - FDA, "Preliminary Finding of No Significant Impact: AquAdvantage Salmon" de 4 de mayo de 2012. De ahora en adelante haremos referencia a este informe como -FONSI provisional 2012. Disponible e internet en el siguiente enlace: http://www.fda.gov/downloads/AnimalVeterinary/DevelopmentApprovalProcess/GeneticEngineering/Ge neticallyEngineeredAnimals/UCM333105.pdf (last ac- cessed on 20 November 2013). Hereafter FONSI. 
Corti Varela, Justo: “Implicaciones de la reciente autorización de comercialización para el consumo humano del salmón modificado genéticamente”. IUS ET SCIENTIA, 2015, Vol. 1, No. 1, pp. 93-109.

DOI: http://dx.doi.org/10.12795/IETSCIENTIA.2015.i01.07

crecimiento aumenta la eficiencia en el uso de instalaciones de piscifactorías a la vez que incrementa la productividad de la cría en cautividad. Esto debería, en principio, aliviar la presión que ejerce la industria extractiva sobre los caladeros naturales en un contexto de crecimiento de la población humana y aumento del consumo de proteínas en los países en desarrollo. Si bien estos elementos positivos pueden deducirse del hecho que el Salmón AquAdvantage tenga un rápido crecimiento, existen otros factores que deben combinarse para alcanzar algunos de ellos. Como veremos a continuación, por ejemplo, el alivio en la pesca extractiva presupone que el Salmón transgénico utilice proteínas no marina en su alimentación. Como esto no es así, al menos hasta ahora, es de proveer que el incremento de productividad conlleve un aumento de piscifactorías de salmones y, con ello, la demanda de harinas de pescado provenientes de capturas extractivas para elaborar los piensos de dichos salmones.

En cuanto a los riesgos potenciales, los podríamos calificar en tres grupos: los vinculados a la seguridad alimentaria, los relativos al medioambiente y los socioeconómicos.

Respecto a la seguridad alimentaria hay que mencionar que todo cambio genético puede, potencialmente, ser causa de toxicidad o alergia. Estos problemas pueden derivar del propio gen insertado, es decir, de la combinación del salmón real y de la anguila marina, algo difícil ya que ambas especies son, en principio, consumidas por el hombre y, por lo tanto, se tienen antecedentes sobre sus efectos. Sin embargo, y también vinculados con los riesgos de alergia y toxicidad, es posible que los nuevos generes interactuen con otros y produzcan efectos que, por separado, nunca se generarían. Este fenómeno es conocido como transgénesis ${ }^{10}$. La evaluación de riesgos realizados por la propia empresa solicitante de la autorización incluye estudios sobre toxicidad y de alergia del propio salmón AquAdvantage, pero no se incorpora información sobre los posibles problemas de transgénesis. Es más, la evaluación definitiva de riesgos de seguridad alimenticia realizada por la FDA $^{11}$ se centra básicamente en las alteraciones de los niveles hormonales y la eventual carácter alergénico del gen incorporado (efectos con son calificados de "directos") y, por otra

\footnotetext{
${ }^{10}$ Para profundizar sobre los posibles riesgos de transgénesis ver Olivier Le Curieux-Belfond, Louise Vandelac, Joseph Caron Gilles-Eric Seralini "Factors to consider before production and commercialization of aquatic genetically modified organisms: the case of transgenic salmon" 12 Environmental Science \& Policy (2009), pp. 170 et sqq., esp. p. 174.

${ }^{11}$ Ver FDA 2015 (op.cit. 6), en p. 66.
} 
Corti Varela, Justo: “Implicaciones de la reciente autorización de comercialización para el consumo humano del salmón modificado genéticamente”. IUS ET SCIENTIA, 2015, Vol. 1, No. 1, pp. 93-109.

DOI: http://dx.doi.org/10.12795/IETSCIENTIA.2015.i01.07

parte, la alteración de la composición de los tejidos biológicos del salmón así como el carácter alegénico de estos cambios (efectos que son calificados de "indirectos"). Más allá de estos riesgos, no se incluyen estudios específicos sobre la interacción con otros genes.

En lo relativo a los riesgos medioambientales, ante la eventualidad de que un espécimen de salmón AquAdvantage escape de su confinamiento, se debe evaluar si el hecho que crezca dos veces más rápido que los salmones convencionales puede constituir una ventaja comparativa decisiva, tanto para el control del territorio como para prolongar sus genes en su descendencia. Es verdad que los salmones criados en cautividad son poco viables en un entorno salvaje. Sin embargo siempre está el riesgo que lleguen a procrear y, sus crías, sí que se encuentren adaptadas para sobrevivir. Por lo tanto el riesgo de entrecruzamiento con salmones libres, colonización de territorios y la perdida de la singularidad genética del salmones salvajes es más que una hipótesis lejana. Para impedir o, al menos, limitar estos riesgos, AquaBouty ha solicitado se limite la autorización de comercialización para:

a) producir los huevos de salmón en sus instalaciones en Canadá.

b) criar los huevos ya fertilizados en cubetas aisladas en una región interior de Panamá.

c) emitir licencias para vender salmones modificados genéticamente a otros criadores de salmones.

Sin embargo, los riesgos de que se genere un escape fortuito nunca se puede eliminar ya que "no single containment measure can be assured to be $100 \%$ effective"12. Por esta razón AquaBounty propuso medidas adicionales que combinan la selección de huevos para garantizar que todos, o casi todos, sean femeninos; con una técnica de esterilización. La primera parte del procedimiento se basaba, inicialmente, en inducir el cambio de sexo de los ejemplares por vía química. En el informe de evaluación ambiental provisional la FDA había destacado que estos procesos de esterilización no son totalmente eficaces y, al menos, un 5\% de los ejemplares puede mantener cierta fertilidad ${ }^{13}$. Para evitarlo se cambió el método de selección a la ginogenesis que sí que

${ }^{12}$ FDA 2010, op.cit. 6, esp. p. 116.
${ }^{13}$ FDA 2010, op.cit. 6, esp. p. 115. 
Corti Varela, Justo: “Implicaciones de la reciente autorización de comercialización para el consumo humano del salmón modificado genéticamente”. IUS ET SCIENTIA, 2015, Vol. 1, No. 1, pp. 93-109.

DOI: http://dx.doi.org/10.12795/IETSCIENTIA.2015.i01.07

garantiza un 100\% de efectividad en la selección de los huevos femeninos, lo que incrementa significativamente el efecto de las técnicas de esterilización ${ }^{14}$.

Más allá de los riesgos medioambientales directos, es decir los ligados a un posible escape, nos encontramos también con riesgos de tipo socio-económicos que pueden tener también un efecto medioambiental indirecto de gran importancia. Un salmón de rápido crecimiento demandará más alimentos, esencialmente obtenidos de harinas de pescado de la pesca extractiva. Para hacernos una idea de la envergadura del problema tenemos que tener en cuenta que cada kilogramo de salmón criado en piscifactorías demanda entre 1,2 y 1,4 kilogramos de pienso seco, que equivale a entre 4 y 5 kilogramos de pescado capturados en mar abierto. Además del problema socioeconómico y medioambiental que esto produce, también se pueden mencionar ciertos efectos no deseados en la distribución de recursos comunes y muy especialmente en la transferencia de los mismos desde países en desarrollo a países desarrollados. Por el precio y calidad del producto la cría de salmón es, en su gran mayoría, destinada a los mercados maduros, es decir a los correspondientes a países desarrollados, o bien a los segmentos de mayor poder adquisitivo de los mercados emergentes. Por el contrario, las capturas necesarias para obtener las cada vez más demandadas harinas de pescado se obtienen en países en desarrollo o, incluso, en lugares donde impera aun la pesca de subsistencia. Así, el salmón de rápido crecimiento puede que incremente uno de los mayores problemas actuales de las piscifactorías, es decir el de la transferencia de recursos proteicos de la periferia al centro.

Los riesgos y beneficios, casi siempre, son de carácter subjetivo y su percepción puede variar de un país a otro. En este sentido hay que decir que, en términos generales el salmón transgénico sigue la lógica hasta ahora preponderante en materia de biotecnología alimentaria: el centro de interés de la investigación está en aumentar la productividad en métodos ya existentes, normalmente vinculados a la cría intensiva, es decir benefician más bien al productor, y no añaden elementos cualitativos que puedan interesar a los consumidores. Es por ello que consideramos que el salmón de rápido crecimiento muy difícilmente pueda ser aceptado en Europa donde la biotecnología alimentaria se percibe como un riesgo innecesario ya que poco aporta, por ejemplo en cuando al sabor, calidad o reducción de impactos medioambientales frente a los

\footnotetext{
${ }^{14}$ Evaluación ambiental definitiva 2015, op.cit. 6, esp. p. 117.
} 
Corti Varela, Justo: “Implicaciones de la reciente autorización de comercialización para el consumo humano del salmón modificado genéticamente”. IUS ET SCIENTIA, 2015, Vol. 1, No. 1, pp. 93-109.

DOI: http://dx.doi.org/10.12795/IETSCIENTIA.2015.i01.07

métodos convencionales de producción. Finalmente, hay que mencionar que el salmón AquAdvantage está protegido por una patente, con lo que incluso los beneficios de productividad puede que, al menos en parte, se transfieran al titular de la patente y no redundarán en precios más reducidos para los consumidores. Esto sin mencionar que, como ya ha ocurrido en la biotecnología agrícola, aumenta la dependencia de los criadores frente a las multinacionales. Recordemos que los primeros ya no podrán controlar el proceso de reproducción y deberán, año tras año, depender de los huevos (estériles) que les vendan las multinacionales biotecnológicas titulares de la patente.

\section{El proceso de evaluación de la FDA}

Los Estados Unidos han desarrollado un procedimiento de siete etapas ${ }^{15}$ acumulativas para evaluar a los animales modificados genéticamente. Las solicitudes tienen que aprobar cada una de las etapas antes de pasar a la siguiente. Como ha ocurrido con los cultivos genéticamente modificados, la evaluación no la realiza una nueva autoridad con competencias exclusivas en materia biotecnológica sino por el marco de agencias federales preexistentes que, antes de la introducción en el mercado de productos biotecnológicos, tenían competencia para revaluar riesgos químicos. Sin embargo, a diferencia de lo que ocurría con las plantas modificadas genéticamente donde las responsabilidades de repartían, según la materia evaluada, entre la EPA Environmental Protection Agency, el Departamento de Agricultura y la FDA, es decir el "Coordinated Framework for the Regulation of Biotechnology”"16, en el caso de los animales modificados genéticamente todo el proceso está a cargo de esta última. Es decir, la FDA evalúa no solo la seguridad alimentaria sino también los riesgos ambientales (tradicionalmente competencia de la EPA). Esto se debe a que los animales transgénicos se consideran “new drugs” y, bajo esta categoría, los evalúa dos comités de la FDA: El Centro para la Medicina Veterinaria, encargado esencialmente de la

\footnotetext{
15 Estas son: 1) La definición del producto; 2) Caracterización molecular del vehículo; 3) Caracterización molecular del animal genéticamente modificado; 4) Caracterización Fenotípica del Animal genéticamente modificado; 5) Durabilidad: Plan Genotípico y Fenotípico; 6) Seguridad alimentaria y medioambiental; y 7) Validación.

${ }^{16}$ Oficina para la Política Científica y Tecnológica - Gabinete de Presidencia de los Estados Unidos “Coordinated Framework for Regulation of Biotechnology”, 51 Fed. Reg. 23,302 (26 de junio 1986), disponible en http://www.aphis.usda.gov/brs/fedregister/coordinated_framework.pdfhttp://www.aphis.usda.gov/brs/fedr egister/coordinated_framework.pdf (último acceso 30 de noviembre de 2015).
} 
Corti Varela, Justo: “Implicaciones de la reciente autorización de comercialización para el consumo humano del salmón modificado genéticamente”. IUS ET SCIENTIA, 2015, Vol. 1, No. 1, pp. 93-109.

DOI: http://dx.doi.org/10.12795/IETSCIENTIA.2015.i01.07

seguridad del animal y de sus productos derivados (incluyendo, en este último caso, la evaluación ambiental); y el Servicio de Inspección para la Salud Animal y Vegetal (APHIS), que se encarga de prever los potenciales efectos sobre la salud de los animales mientras están vivos, es decir, antes de que ser sacrificados y justo después de este momento $^{17}$.

\section{a. ¿Por qué los animales modificados genéticamente son considerados “new animal drugs"?}

De acuerdo con la política de equivalencia sustancial que los Estados Unidos vienen aplicando en materia de organismos genéticamente modificados, estos no se consideran productos diferentes a los ya existentes, por lo que son evaluados según la regulación y autoridades preexistentes. Este sistema obligaba a las tres agencias implicadas (la FDA, la EPA y el USDA) a ampliar el marco de actuación de sus propios estatutos para poder cubrir los productos biotecnológicos con las herramientas jurídicas con que se contaba. Por ejemplo, el maíz Bt (una planta modificada para segregar esporas de un pesticida) fue autorizada al mismo tiempo por la USDA como "plant pest” (en virtud de la Plant Pest Act), por la EPA en calidad de “pesticida” (bajo el mandato de la Federal Insecticide, Fungicide and Rodenticide Act), y por la FDA como “alimento” (bajo la Federal Food, Drug, and Cosmetics Act - FFDCA). Sin embargo, como se adelantara en el párrafo introductorio de este punto, desde la publicación en 2009 de la "Guía sobre animales modificados genéticamente”18 estos animales son regulados solamente por la FDA. Esto es así porque los animales genéticamente modificados se engloban en el concepto de "animal drugs” o drogas animales que, según la FFDCA pueden definirse como "any drug intended foruse inanimals other thanman, including anydrug intended for use in animal feed but not including such animal feed...”19. La FDA ha justificado que esta inclusión debido a que:

\footnotetext{
17 Ver Núria Vàzquez-Salat y otros, op.cit. nota 4, esp. p. 1339.

${ }^{18}$ Center for Veterinary Medicine - FDA, “Guidance for Industry: Regulation of Genetically Engineered Animals Containing Heritable Recombinant Constructs” de 15 de enero de 2009 (revisadas el 17 de mayo de 2011). De ahora en más nos referiremos a este documento como FDA Guidance for Industry.

Disponibles en internet en

http://www.fda.gov/downloads/AnimalVeterinary/GuidanceComplianceEnforcement/GuidanceforIndustr y/ucm113903.pdf (último acceso 30 de noviembre de 2015)

${ }^{19}$ US Federal Food, Drug, and Cosmetic Act, 21 U.S.C. § 321(v).
} 
Corti Varela, Justo: “Implicaciones de la reciente autorización de comercialización para el consumo humano del salmón modificado genéticamente”. IUS ET SCIENTIA, 2015, Vol. 1, No. 1, pp. 93-109.

DOI: http://dx.doi.org/10.12795/IETSCIENTIA.2015.i01.07

"the rDNA construct in a G[M] animal that is intended to affect the structure or function of the body of the $G[\mathrm{M}]$ animal, regardless of the intended use of products thatmay be produced by the $\mathrm{G}[\mathrm{M}]$ animal, meets the FFDCA drug definition.”20

En otras palabras, la construcción de ADN recombinante es considerar un elemento no alimenticio que pretende afectar o modificar la estructura de una animal de modo similar a una droga. Por ello es considerada una "nueva droga animal”, es decir, una droga que no ha sido utilizada en la práctica ni se encuentra aún reconocida por la comunidad científica y, por lo tanto, todavía se desconoce si es segura y efectiva para su uso en animales ${ }^{21}$.

De acuerdo con la New Animal Drug Application (NADA) de la FFDCA, la evaluación de una droga animal debe seguir tres criterios: (a) la seguridad animal, (b) la efectividad de la droga y (c) la seguridad humana. En principio la NADA solo incluye la evaluación de riesgos sobre "the

health of man or animal"22, excluyendo por lo tanto el impacto mediambiental en sentido extensor. Esta idea se corresponde con la de una evaluación de riesgos centrada en una “droga”. Sin embargo, debido a los múltiples comentarios críticos que se recibieron durante el período de consulta pública de $2010^{22}$, la FDA ha considerado oportuno incluir la evaluación de "environmental effects that directly or indirectly affect the health of humans or animals”23. Esta ampliación en el campo de evaluación de riesgos no incluye, de todos modos, los riesgos puramente medioambientales, por ejemplo el impacto sobre la biodiversidad oceánica en caso de un escape. Estos últimos tampoco podrían ser evaluados por la EPA, probablemente la agencia con mayor experiencia en la materia pero también muy proclive a realizar análisis muy

\footnotetext{
${ }^{20}$ FDA Guidance for the Industry, op.cit. nota 18, esp. en p. 6.

${ }^{21}$ Para una explicación más detallada ver George Kimbrell y otro, op.cit. nota 1.

${ }^{22}$ Ver, por ejemplo, la carta firmada por 29 congresistas pidiendo a la FDA "stop the approval process immediately" debido a los potenciales riesgos sobre la salud y el medioambiente que nunca habian sido debidamente evaluados. Ver Carta de Peter DeFazio, U.S. Representative, et al., to Margaret A. Hamburg, FDA Commissioner, 29 de septiembre de 2010, disponible en el sitio de internet http://www.defazio.house.gov/ index.php?option=com_content\&task=view\&id=629 (ultimo acceso el 30 de noviembre de 2015).

${ }^{23}$ Oficina para la Política Científica y Tecnológica - Gabinete del Presidente de los Estados Unidos "Case Study $\mathrm{n}^{\circ}$ 1: Growth-Enhanced Salmon" (2001), disponible en el sitio de internet http://www.whitehouse.gov/galleries/Issues/ceq_ostp_study2.pdf (último acceso 30 de noviembre de 2015)
} 
Corti Varela, Justo: "Implicaciones de la reciente autorización de comercialización para el consumo humano del salmón modificado genéticamente”. IUS ET SCIENTIA, 2015, Vol. 1, No. 1, pp. 93-109.

DOI: http://dx.doi.org/10.12795/IETSCIENTIA.2015.i01.07

pormenorizados, ya que de acuerdo con las distribuciones de competencias decididas para los animales genéticamente modificados no tendría capacidad para ello²4.

\section{b. La evaluación de seguridad alimentaria}

A diferencia de lo que ocurre con la presunción de seguridad que gozan las plantas modificadas genéticamente, las “new animal drugs” no se incluyen en la definición de “Generally Recognized as Safe” (GRAS). Como consecuencia de ello, se debe realizar una evaluación riesgos completa y no sólo sobre aquellos aspectos sobre los que versa la modifiacción genética. Al igual que ocurre con las plantas transgénicas en Europea, es el solicitante quien debe realizar la evaluación, es decir, la empresa interesada en obtener la aprobación. La agencia evaluadora, en este caso el Centro para la Medicina Veterinaria de la FDA, brinda asesoramiento sobre cómo debe realizarse la evaluación y controla que la misma se haya desarrollado según los estándares de seguridad deseables.

En su informe de 2010, la agencia ya había declarado que el salmón AquAdvantage era "as safe as food from conventional Atlantic salmon" 25 , y ha vuelto a arribar a la misma conclusión en su informe definitivo de $2015^{26}$. En ambos casos para llegar a esta conclusión la empresa solicitante comparó salmones genéticamente modificados con otros convencionales para evaluar si había diferencias en cuanto a sus características nutricionales. En ningún caso se realizaron estudios sobre la interacción que podría tener el ADN recombinante con otros genes de los salmones. Tampoco se evaluó la estabilidad molecular de la modificación más allá de las 7 generaciones a las cuales se limitó el grupo de especímenes estudiados ${ }^{27}$.

\section{c. La evaluación de riesgos medioambientales}

\footnotetext{
${ }^{24}$ Michael Bennett Homer “Frankenfish . . . It's What's for Dinner: The FDA, Genetically Engineered Salmon, and the Flawed Regulation of Biotechnology" 45 Columbia Journal of Law and Social Problems (2012), pp. 83 et sqq. esp. p. 105.

${ }^{25}$ FDA 2010, op.cit. en nota 7, esp. p. 71.

${ }^{26}$ FDA 2015, op.cit. en nota 6, esp. p. 107

${ }^{27}$ FDA 2015, op.cit. en nota 6, esp. p. 14.
} 
Corti Varela, Justo: “Implicaciones de la reciente autorización de comercialización para el consumo humano del salmón modificado genéticamente”. IUS ET SCIENTIA, 2015, Vol. 1, No. 1, pp. 93-109.

DOI: http://dx.doi.org/10.12795/IETSCIENTIA.2015.i01.07

En relación con los riesgos ambientales, hay que señalar que la FDA carecía de experiencia en la evaluación de este tipo de riesgos ${ }^{28}$. Sorprendentemente, durante la evaluación del AquAdvantage la FDA no solicitó asesoramiento ni a la EPA ni a la USDA, a pesar de las múltiples recomendaciones realizadas por un panel de expertos en biología marina tal como recoge la consulta pública de 2010.

Como ya se mencionó en el tercer punto de este artículo, los riesgos de sufrir un escape del lugar confinamiento y, eventualmente, convertirse en una especie invasiva, se encuentra en el centro de las preocupaciones de la evaluación medioambiental de riesgos. Si bien AquaBounty no niega la existencia del riesgo, propone varias medidas para mitigarlo. La primera es la ya mencionada selección de huevos femeninos para luego esterilizarlos. La segunda, el confinamiento en piscinas localizadas en un entorno tropical ya que, en caso de escape, y ante la rara posibilidad de que un espécimen lograse alcanzar un río o curso de agua natural, la temperatura del agua exterior sería tan alta que no permitiría la supervivencia del individuo. Estas dos propuestas son la clave de la autorización otorgada por la FDA tanto en el FONSI preliminar de 2012 como en el definitivo de $2015^{29}$.

Sin embargo, muy poca atención se le brindado a los riesgos derivados del traslado de los huevos desde el lugar de reproducción, en Canadá, al lugar de cría, en Panamá. Resulta interesante resaltar que sólo se menciona la obligación de etiquetar las bolsas de transporte con textos bilingües español-inglés y otras condiciones de mantenimiento de temperatura ${ }^{30}$. Probablemente lo más sorprendente es que la FDA, si bien explica detalladamente cómo deben ser las infraestructuras de reproducción y cría en Canadá y Panamá, respectivamente, reconoce que no podrá evaluar directamente la ejecución de dichas recomendaciones y/o condiciones ya que las instalaciones se encuentran en lugares fuera de su jurisdicción, salvo

"insofar as it was necessary to do so in order to determine whether there would be significant effects on the environment of the United States due to the

\footnotetext{
${ }^{28}$ Paul Voosen, “Panel Advises More Aggressive FDA Analysis of Engineered Salmon”, New York Times, artículo publicado el 21 de septiembre de 2010, disponible en http://www.nytimes.com/gwire/2010/09/21/21greenwire-panel-advises-moreaggressive-fda-analysis-of71171.html?pagewanted=all (ultimo acceso el 20 de noviembre de 2015).

${ }^{29}$ FONSI definitivo 2015, op.cit. nota 6.

${ }^{30}$ Evaluación Ambiental definitiva 2015, op.cit. nota 6, esp. p. 70.
} 
Corti Varela, Justo: “Implicaciones de la reciente autorización de comercialización para el consumo humano del salmón modificado genéticamente”. IUS ET SCIENTIA, 2015, Vol. 1, No. 1, pp. 93-109.

DOI: http://dx.doi.org/10.12795/IETSCIENTIA.2015.i01.07

origination of exposure pathways from the production facilities in Canada and Panama"31.

En consecuencia, será necesaria la colaboración de autoridades ambientales locales para poder comprobar el impacto local de la actividad y evaluar la posible inclusión de medidas compensatorias o paliativas complementarias ${ }^{32}$.

Finalmente, algunos autores ${ }^{33}$ han señalado que el salmón atlántico es una especie protegida por la Endangered Species Act (EAS), lo que obligaría a realizar una evaluación de impacto ambiental total sobre territorio americano siguiendo los estándares del muy estricto US Fish and Wildlife Service (FWS) y de la National Oceanic and Atmospheric Administration (NOAA).

\section{d. La transparencia a que fue sometida la evaluación}

No se puede negar que la autoridad americana no haya sometido el proceso a no una sino tres períodos de consulta pública. El primero fue justo después de la publicación de las Guías para la Industria en 2009, y los otros dos a continuación de los informas de la FDA sobre el salmón AquAdvantage en 2010 y 2012. Los tres períodos tuvieron una duración de 60 días y como consecuencia de ellos se incorporaron al proceso de toma de decisiones un gran número de comentarios, alrededor de 30.000 por cada período. Cada período consulta tuvo como respuesta un documento de la FDA en donde se resumían y reagrupaban los principales comentarios enviados y, a la vez, se respondía a estos con los argumentos que la FDA consideraba adecuados.

Algunos autores ${ }^{34}$ han insinuado que estos resúmenes han sido demasiados sintéticos y que las respuestas ofrecidas por la FDA fueron vagas y generales. Sin embargo no se puede negar que el mecanismo ha funcionado relativamente bien y que ha obligado a la FDA no solo a argumentar mejor sus decisiones sino que también, incluso, a aumentar las medidas de transparencia del procedimiento. Por ejemplo, a raíz

\footnotetext{
${ }^{31}$ FONSI definitivo 2015, op.cit. nota 6, p. 3/4.

32 En este sentido ver Canadian Science Advisory Secretariat "Summary of the Environmental and indirect human health risk assessment of Aquadvantage Salmon” Science Response 2013/023. Disponible en internet en: http://www.dfo-mpo.gc.ca/csas-sccs/Publications/ScR-RS/2013/2013_023-eng.pdf (último acceso 30 de noviembre de 2015).

${ }^{33}$ Ver, por ejemplo, Núria Vàzquez-Salat y otros, op.cit. nota 4, esp. p. 1337.

${ }^{34}$ Michael P. McEvilly "Lack of Transparency in the premarket approval process for Aquadvantage

Salmon” 11 Duke Law \& Technology Review (2013), pp. 413 et sqq.
} 
Corti Varela, Justo: “Implicaciones de la reciente autorización de comercialización para el consumo humano del salmón modificado genéticamente”. IUS ET SCIENTIA, 2015, Vol. 1, No. 1, pp. 93-109.

DOI: http://dx.doi.org/10.12795/IETSCIENTIA.2015.i01.07

de la experiencia adquirida se decidió crear "public advisory committee meetings” antes de tomar cualquier decisión favorable a la aprobación de un animal modificado genéticamente.

Si bien el público y los principales interesados han tenido muchas oportunidades de introducir su visión en procedimiento, tal vez la principal debilidad de los períodos de consulta radica en las dificultades para el acceso a la información relevante. Antes de tomar cualquier decisión sobre la aprobación de un "new animal drug" la Trade Secrets Act prohíbe a la FDA revelar cualquier información relevante que pueda ser considerada secreto comercial del solicitante. Es verdad que el Comisionado puede preparar, bajo su responsabilidad y buen criterio, un resumen con una selección de datos sobre la seguridad y efectividad del producto para, así, informar al público general durante el proceso de consulta, por ejemplo, antes de las reuniones del comité asesor (public advsory committee meetings). Así se hizo con el salmón AquAdvantage y, aunque el contenido del resumen fue criticado por sus limitaciones, en realidad la mayor parte de las quejas se refirieron a lo exiguo los plazos. El resumen se publicó sólo dos semanas antes de las reuniones, con lo cual los interesados prácticamente no tuvieron tiempo para analizar en detalle la información, consultar con expertos, o investigar alternativas con las que elaborar preguntas sólidas a los miembros del comité. A pesar de ello, y aunque pueda ser mejorable, no puede negarse que la FDA ha realizado un ejercicio muy importante de transparencia durante el proceso.

\section{Conclusiones}

La autorización del salmón AquAdvantage es, sin duda, un evento clave en el proceso de toma de decisiones sobre productos biotecnológicos ya que constituye el primer animal modificado genéticamente en ser autorizado para comercializarse con fines de alimentación humana.

Si el consumidor americano acepta el salmón AquAdvantage probablemente se abra la puerta a nuevas autorizaciones que, sin duda, cambiarán por completo el campo de las relaciones entre derecho y ciencia, al menos en cuanto a biotecnología se refiere.

Para lograr la aceptación del público es necesario no solo demostrar que la agencia encargada de la evolución de riesgos, tanto sanitarios como medioambientales, ha realizado un trabajo pormenorizado y completo, sino también que el procedimiento 
Corti Varela, Justo: “Implicaciones de la reciente autorización de comercialización para el consumo humano del salmón modificado genéticamente”. IUS ET SCIENTIA, 2015, Vol. 1, No. 1, pp. 93-109.

DOI: http://dx.doi.org/10.12795/IETSCIENTIA.2015.i01.07

ha tenido la suficiente transparencia como para permitir inputs y respuestas de las diferentes sensibilidades de la sociedad.

En este sentido, el hecho que la evaluación de riesgos ambientales la haya realizado una agencia con poca o ninguna experiencia en el ámbito medioambiental no ayuda a convencer al publico en general sobre la pertinencia y seriedad del proceso. Además, el proceso de consulta pública ha demostrado que en la sociedad americana hay un rechazo mucho más fuerte que el inicialmente imaginado contra los animales genéticamente modificados destinados a la alimentación humana. Es más, varias cadenas de supermercados como Trader Joe's, Aldi y Whole Foods han dicho que iniciarán un boicot contra el salmón AquAdvantage para garantizar que éste no se venda en sus tiendas.

Una medida que podría limitar el efecto de este rechazo sería el establecimiento de un etiquetado obligatorio sobre el salmón modificado genéticamente, similar al que impera en la UE para las plantas transgénicas. El etiquetado permitiría distinguir entre productos convencionales y biotecnológicos y dejaría que los consumidores la elección sobre qué comprar. Sin embargo, siguiendo una larga tradición, las autoridades americanas se niegan a ello aduciendo que los alimentos modificados genéticamente son equivalentes a los convencionales y, por lo tanto, no resulta necesario distinguirlos.

El etiquetado y la trazabilidad se impondrá, sin lugar a dudas, para la producción destinada a la exportación, particularmente a la UE, donde los animales modificados genéticamente no tienen visos de ser autorizados. Aunque ni siquiera se haya presentado una solicitud de autorización, las guías de evaluación de riesgos de animales modificados genéticamente de la Agencia Europea de Seguridad Alimentaria (EFSA) son mucho más estrictas que el procedimiento de "new animal drugs" de la FDA, por lo probablemente el salmón modificado genéticamente lo tendrá mucho más difícil en el viejo continente. Sin mencionar el fuerte rechazo, casi unánime, que la sociedad europea manifiesta contra los animales genéticamente modificados para consumo alimenticio. Sin embargo, esta última cuestión escapa al objeto de estudio del presente artículo y, esperamos, poder abordarlo en otra contribución. 